\title{
Effective Distribution of Large Scale Situated Agent-based Simulations
}

\author{
Omar Rihawi, Yann Secq and Philippe Mathieu \\ LIFL (CNRS UMR 8022), Lille 1 University, Villeneuve d'Ascq, France
}

Keywords: Distributed Multi-agent Simulations, Flocking Behaviour, Prey-predator Model.

Abstract: $\quad$ Agent-based simulations have increasing needs in computational and memory resources when the the number of agents and interactions grows. In this paper, we are concerned with the simulation of large scale situated multi-agent systems (MAS). To be able to simulate several thousands or even a million of agents, it becomes necessary to distribute the load on a computer network. This distribution can be done in several ways and this paper presents two specific distributions: the first one is based on environment and the second one is based on agents. We illustrates the pros and cons of using both distribution types with two classical MAS applications: prey-predator and flocking behaviour models.

\section{INTRODUCTION}

Agent-based simulations are used by researchers to provide explanations about real life phenomena like flocking birds behaviour or population co-evolution. The interesting aspects of such approach is that interactions at individual level (microscopic level) leads to emerging patterns at a global level (macroscopic level). In these types of phenomena, the simulation is made of agents that are situated in an environment and interact together to achieve the necessary macroscopic level. Agents (Wooldridge and Jennings, 1995; Russell and Norvig, 1996) are autonomous entities that observes their environment and acts upon it by following their own goals. Agents behaviours can range from purely reactive agents to cognitive agents, which can involve planning and learning abilities. In our study, the environment is the mediation layer that allows a spatial arrangement of agents and can handle agents interactions. Thus, we restrict our study to these kind of spatial based simulations (Cosenza et al., 2011) where agents can only interact when they are close to each other.

Unfortunately, when the number of agents or interactions grows in such simulations, resources in computing costs and memory can rapidly exceed the capacity of a single computer, it then becomes necessary to distribute the load on a set of computers. Nevertheless, load distribution is not an easy task when its dynamic, and evolution is strongly linked to agent behaviour complexity and to agent movements within the environment.

This paper is focused on large-scale situated multi-agent simulations and problematic issues linked to the distribution of multi-agent simulators like: time management, agents migration (Motshegwa and Schroeder, 2004) and load balancing. Time management is an important problem that many researchers investigate. Several models have been proposed: a single global logical time step for the system or multiple time steps (Scerri et al., 2010; Siebert et al., 2010). Earlier works have been done on Virtual Time (Jefferson, 1985) which explain it for discrete event simulations and multi-agent system (MAS) can be considered as discrete event simulation, if we consider an interaction as an event. Load balancing (Yamamoto et al., 2008; Logan and Theodoropoulos, 2001) is also an important issue in any distributed system, if the load between machines is not similar, the distribution will not be efficient especially for the initial state. We believe that the main concepts of multi-agents system (agents and environment) must be taken into account when we distribute some kind of applications to avoid any disproportionate load. For that, we study these issues on two distribution schemes: the repartition of the environment and the repartition of the agents list. We have developed a prototype distributed simulator that can handle both distributions types and we have experimented them on two classical situated agentbased models.

The next section details related works in the field of distributed multi-agent simulators. The third section presents the two distribution types evaluated in this paper. The fourth section describes our prototype platform that manages these two distribution types, while the fifth section provides experimentations re-

312 Rihawi O., Secq Y. and Mathieu P.

Effective Distribution of Large Scale Situated Agent-based Simulations. DOI: 10.5220/0004756903120319

In Proceedings of the 6th International Conference on Agents and Artificial Intelligence (ICAART-2014), pages 312-319

ISBN: 978-989-758-015-4

Copyright (C) 2014 SCITEPRESS (Science and Technology Publications, Lda.) 
sults that have been gathered on two applications: the prey-predator model and flocking behaviour model.

\section{RELATED WORK IN DISTRIBUTED MAS}

Even if some MAS simulations platforms are able to distribute their computation, large-scale MAS simulators are not yet mainstream and are still under active research. The Repast-HLA (Kuhl et al., 1999) provides components to build multi-agent simulations on a network with a shared middleware. The main advantage of this approach is the transparent migration from the centralized to decentralized runtimes, but all optimizations are done at the virtual machine level and thus, cannot be easily tweaked by simulators developers. It should also be noted that the HLA approach is more fitted to coordinate heterogeneous simulation engines than to gain speedup in a large scale simulations.

Other interesting works are D-MASON (Cordasco et al., 2011) and the AglobeX (Šišlák et al., 2009) platform. D-MASON is an extension of the $M A$ $S O N$ toolkit to allow its distribution on a network. $D-M A S O N$ is based on a master/workers approach, where the master assigns a portion of the whole computation (like a set of agents) to each worker. Then, for each simulation step, each worker executes agents behaviours and communicates its result to all interested workers.

AglobeX is also built on the same master/slave pattern and both platforms use simple applications: airplanes simulation for AglobeX and a flocking behaviour for D-MASON. These applications are interesting but they do not imply complex or conflicting interactions. Indeed, others applications like preypredator introduce such interactions (two predators attacking the same prey) that have to be handled gracefully through some tie-break mechanisms.

Another interesting platform is GOLEM (Bromuri and Stathis, 2009) which uses Ambient Event Calculus language to define simulations involving cognitive agents. This platform relies on the notion of container that represent a simulator that can be distributed on a computer. Containers can be nested to allow the definition of complex hierarchies.

In all above platforms, there is no capability given to the user to define and control the distribution process (see table 1). Our goal is exploring different distribution types and providing the ability for the user to choose the most suitable type according to his application domain. For that, we develop our own prototype in order to evaluate the pertinence of our work.

\section{MAS DISTRIBUTION TYPES}

To achieve large-scale simulations with a high number of agents and interactions, the distribution on a computer network of the simulator becomes necessary. In this paper, we study two different types of distribution (figure 1). The first one, that we call agents distribution, consists in keeping one global environment shared by all agents and to distribute agents behaviours computations on several machines. The second one, that we call environment distribution, the environment is divided in several slices and these slices are distributed between machines. Depending on the application that is simulated, we believe that one distribution scheme will be more adapted than the other. The following paragraphs detail the agent and environment distribution:

With the agents distribution, each machine handles a part of the agents list and can communicate with other machines if any changes need to be made on the environment. This type of distribution should be fitted for simulations involving cognitive agents, or agents whose behaviour requires intensive computations. The main issue with this approach is agent repartition (Miyata and Ishida, 2008), particularly when agents are dynamically created or destroyed during a simulation. However, each machine communicates with others for collecting needed information about other agents which exist on other machines (we call this ghost-agents)

With the environment distribution, the environment is sliced in parts and each slice is distributed on a machine with its agents. In this approach, the environment is no more global and thus a specific protocol to allow agents' migrations from one environment slice to the other has to be defined. We also need to take care of the situation when two agents situated on different environment slice need to interact. However, a specific communication protocol has to be defined to allow exchanging information between machines about agents close to the edge-zone on environment borders.

To handle this information exchange and to allow agents' interactions even when they are located on distinct machines, we introduce a ghost area mechanism (figure 2) that defines an area around the edgezone environment slice, which is transmitted from neighbouring machines at each simulation time-step (TS). This area consists of separated environment parts on different machines, which represents the state of neighbouring parts as a ghost area (not a real area). This area will be updated and informed with all changes by one-shoot-message each time that agents want to interact. As we can see in figure 2, each ma- 
Table 1: Comparison between platforms.

\begin{tabular}{|l|l|l|l|l|}
\hline Platform & NbOfAgents & NbOfMachines & Model & Distribution types \\
\hline Repast & 68 billions & HPC-32000 cores & Triangles (simple) & Only one type \\
\hline DMASON & 10 millions & 64 & Boids (simple) & Only one type \\
\hline AglobeX & 6500 & $6(22$ cores) & Airplanes (simple) & Only one type \\
\hline GOLEM & 5000 & 50 & Packet-World (complex) & Only one type \\
\hline Our testbed & 100 millions & 200 & Prey-predator (more complex) & Two types \\
\hline
\end{tabular}
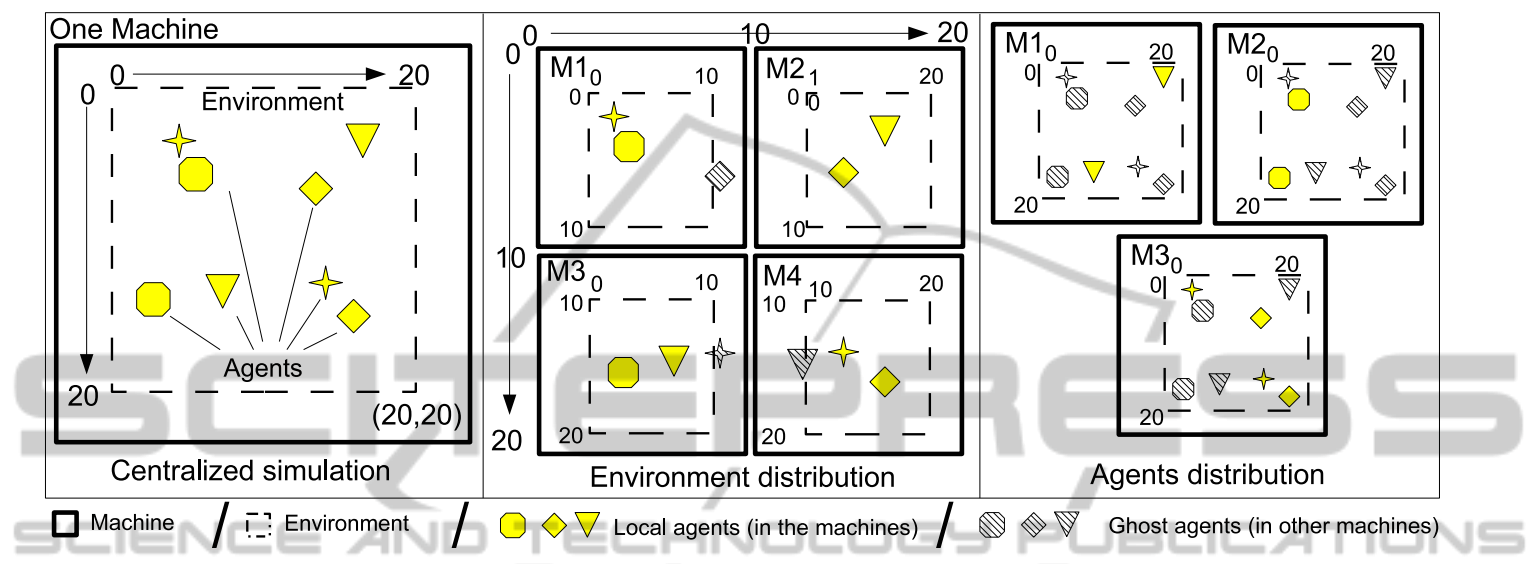

Figure 1: A centralized simulation can be distributed by two different ways: Environment Distribution or Agent Distribution.

chine has to receive ghost-areas from neighbours and also has to send ghost-areas for others too. Ghost area approach is similar to ghost data which is used with some researchers for visualize parallel simulation (Isenburg et al., 2010).

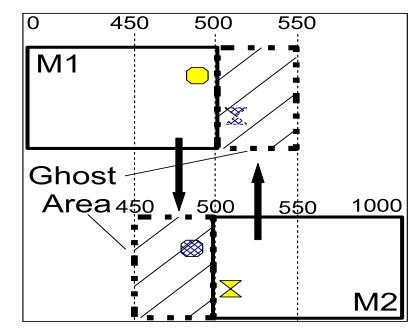

Figure 2: Exchange information between two machines for ghost areas (each machine has a surrounding ghost-area from others).

\section{DISTRIBUTED SIMULATOR DYNAMIC}

We focus in this paper on two distribution types: the environment distribution and the agents distribution. To implement these two distribution types, we have developed a prototype with the Java language. The user can choose which type of distribution he wants according to his application. In our framework, machines run with a simple broadcast communication layer, where each machine can reach other machines.
Each machine makes a part of the calculation during each time step (TS) and it communicates with others to build a complete global simulation view. More precisely, our framework is divided into simulation parts, each simulation part manages one environment part with its agents in case of environment distribution or manage a list of agents in case of agents distribution (figure 1). Each simulation part consists of three layers: a communication layer that establishes connections with others and it is responsible for exchange messages, a simulator layer that shares information and transfers agents and an application layer where agents are defined and are able to interact with each others. In each time step (TS), the simulator gather all interactions that agents wish to execute, then analyses that no conflicting interactions happens (otherwise, a tie-break rule defines which interaction succeeds and which one fails) and apply all interactions. Then, the simulator has to wait for notifications from other machines before going to the next TS.

The simulation is divided in two main stages, the initialization stage and the running stage:

- initialization: with environment distribution, the first step is to divide the environment into different slices on different machines. That can be through a configuration file containing lines with a machine ID, the machine name or IP, the environment slice ranges and the initial agents contained within that slice. In case of agents distribution, the user divide agents between machines in the con- 
Environment distribution file:

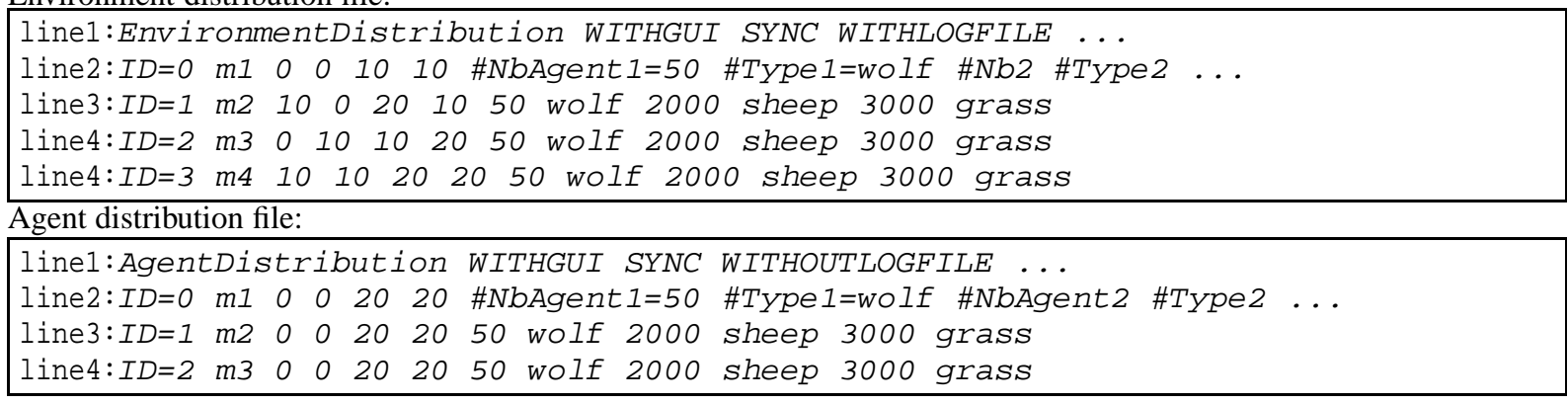

Figure 3: An example of configuration txt-files according to the figure 1.

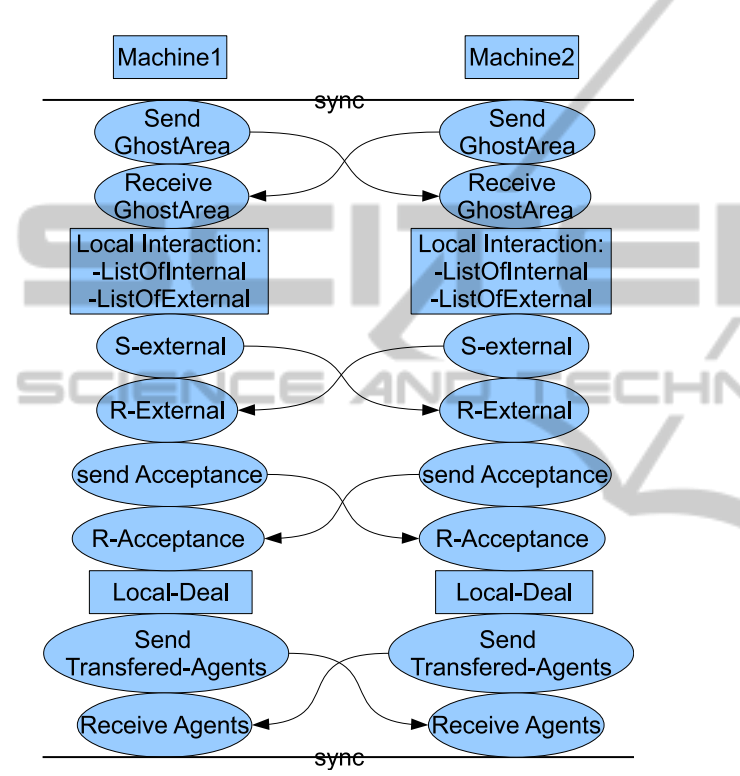

Figure 4: Decomposition of a time step (TS) execution.

figuration file (see figure 3).

- running: during execution, each machine has to collect information from neighbouring machines about ghost-area information in case of environment distribution or information about other agents in case of agents distribution. Then, the simulator asks each agent about its next interaction. However, and in case of environment distribution, if there are agents that cross an edge-zone, they have to be transferred to another computing machine. In case of agents distribution, there is no agent mobility between machines, unless some load-balancing has to be done.

To be more precise about the dynamic of a simulation step, figure 4 illustrates the main loop that are applied in the context of two machines.

We first describe all steps for the environment distribution approach (as illustrated in figure 4), then we briefly address what is different for agents distribution:
- Each machine sends its ghost areas to neighbouring machines.

- Each machine waits to receive ghost areas from its neighbours.

- Each simulator within each machine gathers interactions from its agents and creates two lists: internal interactions list (between agents on the same machine) and external interactions list (between agents that exist on different machines). Internal interactions could be evaluated directly by the simulator, while external interactions cannot be evaluated directly because they require some communication to reach an agreement with other machines in order to avoid conflicting interactions (when two wolves want to eat the same sheep for example).

- Each machine sends its external interactions to other related machines and waits for agreements or rejections of interactions. If an interaction is refused, agents can re-ask for other interactions. However, it can be implemented as a loop between machines until all agents are satisfied (all machines agrees). Normally, it could not be more than 2 or 3 messages loop that can be exchanged between machines, but to avoid this loop we simply drop the interaction if it is refused in this time step of the simulation.

- After that, all interactions have been resolved and each machine executes its interactions.

- Then, each machine checks if some agents have to be moved outside of their environment slice and if it is the case, theses agents are transferred to neighbouring machines.

- Finally, all machines are ready to visualize their environment slices and wait for the synchronization barrier before moving to next TS.

In the agent distribution context, the steps are similar, but instead of exchanging ghost area information, the information about agents that are sent and 


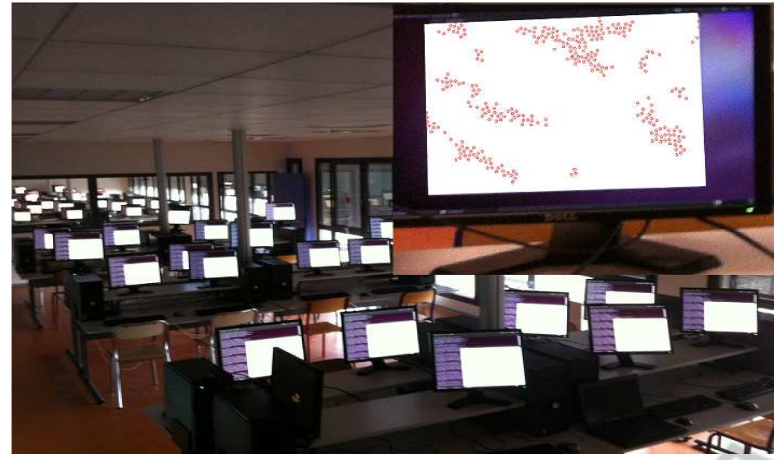

Figure 5: Five million agents on 50 machines.

no agent transfer occurs (unless some load-balancing is taken into account).

Figure 5 shows our testbed scalability with 50 machines to simulate the flocking birds behaviour (Reynolds, 1999). Each machine has 100000 agents and the simulation has $50 \times 100000=5$ million agents in total.

\section{EXPERIMENTATIONS}

This section details experimentations that have been realized in order to evaluate our platform scaling. Two applications have been studied: prey predator (Wilensky, 1997) and a flocking models (Reynolds, 1999).

Prey Predator: which is a classical MAS simulation using agents with goals. A predator is an organism that eats another organism (the prey). For example of predator and prey, we can simulate the co-evolution of wolves and sheep. Predators and preys evolve together, the prey is a part of the predator's environment, and the predator dies if it does not get enough food (prey). Also, the predator is a part of the prey's environment, and the prey dies if it is eaten by the predator. The fastest predators in the environment are able to catch food and eat, so they survive and reproduce, and make up more and more of the population. The fastest preys are able to escape from predators, so they survive and reproduce, and make up more and more of the population. An example of this model is wolf-sheep-grass model (Wilensky, 1997).

Flocking. This simulation illustrates a steering behaviour that commonly observed with birds (Reynolds, 1999) (or fish) that evolves in groups. In this model there is only one kind of agent (like bird) which can move forward with a group of other near birds, which are in its perception range. Normally in this simulation and after some iterations, groups of agents are emerging and after some times, there is only one big group of birds that moves smoothly together. On the environment's view, there is non homogeneous distributions, some parts of the sky hold a lot of birds, while others are less filled.

These applications have been chosen because they imply distinct population dynamics. Indeed, in a prey predator model, prey and predator are moving but they are homogeneously distributed within the environment, while the flocking model, even if the distribution is homogeneous at the beginning of the simulation, rapidly flocks emerge and after some times, only one main flock appears. It means that these two applications illustrate the trade-off that has to be made while distributing the load across a computer network, and the type of distribution can give really different results.

\subsection{Experimentations Description}

All the following experiments have been done on homogeneous hardware with a basic Linux PCs network (Intel-R CoreTM2 Duo CPU E8400 3.00GHz, memory $4 \mathrm{~GB}$ and $100 \mathrm{Mb}$ connection). In these experiments, several initial configurations have been set:

- We are able to simulate a large-scale experiments on 50 machines with 5 million agents (see figure 5). But to facilitate the performance measuring, some experiments are ranging from 1 up to $16 \mathrm{ma}-$ chines.

- At the beginning of the simulation, the number of agents which is usually used: for prey-predator (Wolf-Sheep-Grass model) 5000 agents per machine, for flocking (Birds) 10000 Agents per machines. That means, if we have one experience with 2, 4, 8 and 16 machines, we use 5000x $16=$ 80000 agents for $16,8,4$ and 2 machines to make a reasonable comparison between machines in this experimentation.

- For the agent distribution list, agents are divided equally between machines.

- For the environment distribution, environment are divided equally between machines as a grid as possible (see figure 1).

- Perceptions of all agents are small with respect to the environment slices that are used, and the size of ghost areas has been chosen as the maximum agent perception to avoid any privation of any agent.

\subsection{Scaling the Platform from 9 to 50 Machines}

We have tested a wolf-sheep-grass model with initial 250000 agents distributed on 9, 16, 25 and 50 ma- 


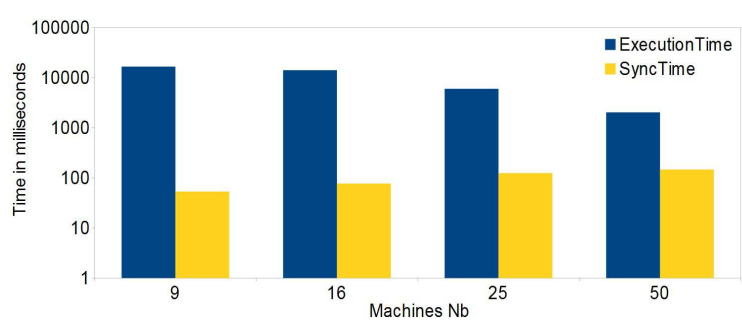

Figure 6: TS delay \& communication delay in prey-predator model with environment distribution approach.

chines by environment distribution approach. Figure 6 shows that the execution time is significantly reduced when more machines are used. In this figure, the first column represents the whole execution time, while the second one is the synchronization delay. The figure shows that with small number of machines, the synchronization delay is small and the execution time is large as the number of agents is large too. Whereas, if the number of agents small and the number of machines is larger (in case of 50 machines), the synchronization delay has to be large too, but the execution time is small. It is clear that, it will not be efficient to distribute small number of agents on large number of machines or large number of agents on small number of machines.

\subsection{Effective Distribution of a MAS Simulation}

Next experiments evaluate the performance of our distributed simulation with two types of distribution: environment distribution and agents distribution, and on two models: Flocking and prey-predator.

Figures 7 and 8 show results for two types of distributions (agents and environment) for each applications. Figure 7 shows that in flocking model and in case of agents distribution the performance is better than environment distribution. That is because, in case of environment distribution there are huge groups of birds that can fly together and swap between machines. That can increase the execution time as there are more charge of birds on one machine (one environment part) than others. Whereas, in case of agent distribution we have the same number of agents on each machine and the execution time will be the same for all. Figure 8 shows that prey-predator model has completely opposite action than flocking model, the execution time is better in case of environment distribution type than agent one, that is because there are no huge groups of agents on the same patch of environment and swap between machines (like the case of flocking). In prey-predator model, agents can reproduce and die during the simulation time steps, for

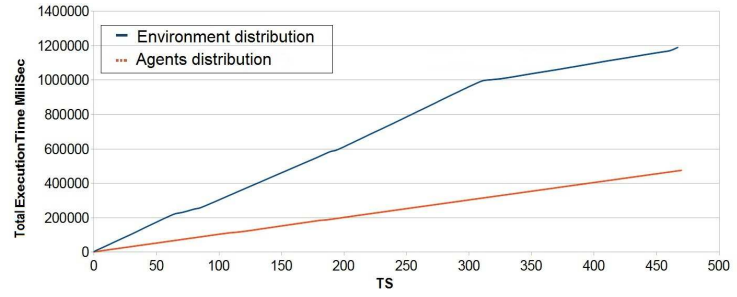

Figure 7: Total execution-time of flocking model with two different distribution types.

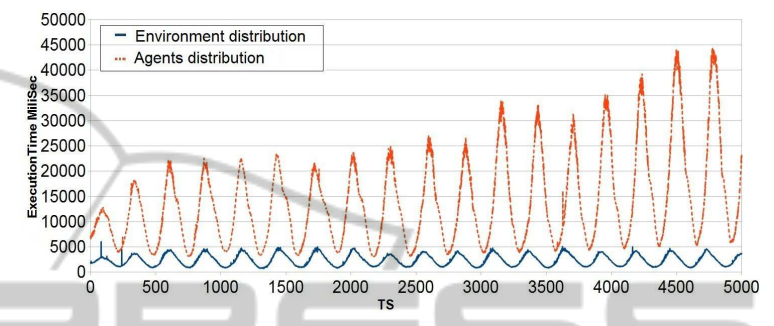

Figure 8: Execution-Time of prey-predator model with two different distribution types.

that the execution time looks like a cosine function as the number of agents is reduced and re-increased during the simulation.

To summarize, environment distribution type is better for prey-predator model than agents distribution one, whereas agents distribution type is better for flocking model than environment one.

\subsection{Communication Costs Evaluation}

In this experimentation, we evaluate the volume of messages exchanged in our two applications. We test the flocking and wolf-sheep-grass models on two machines with environment distribution approach. Figure 9 shows that the flocking model has important variations in messages volume, while prey-predator model did not have such peaks and is more stable. These differences come from the agent behaviour: in flocking model, we may have a huge number of birds moving from one machine to another and that means bigger messages to transfer these agents and so more communication costs. Whereas in prey-predator

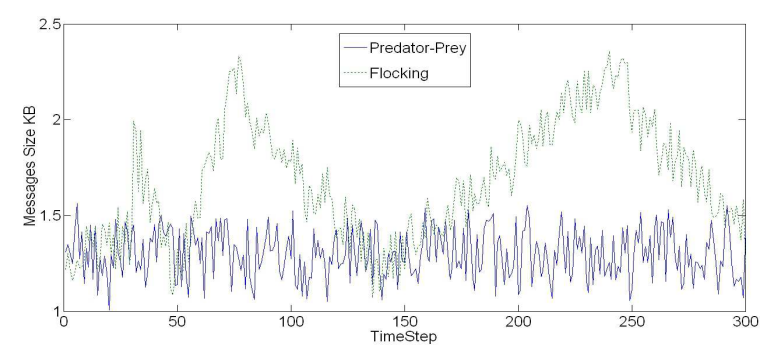

Figure 9: Communication costs of 300 TS in environment distribution approach. 


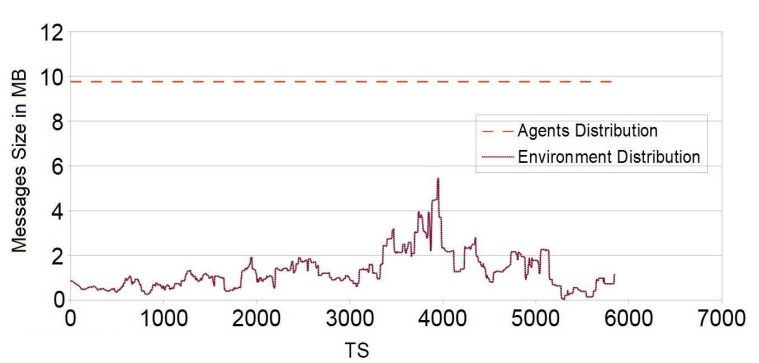

Figure 10: Messages size of flocking simulation.

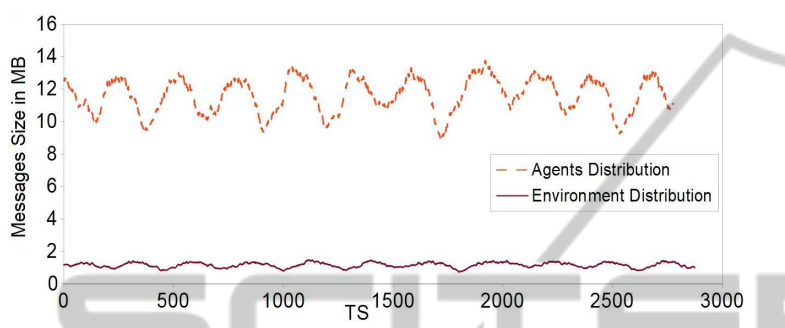

Figure 11: Messages size of prey-predator simulation.

model, it is not the case because agents do not have group behaviours like in the flocking, so the model is more stable in messages exchanged between machines. This figure demonstrates that in distributed multi-agents simulations with spatial environments, speed up is highly related to the dynamic of agents movements and agents models.

To detail more flocking behaviour, we test communication cost on both distribution types, figure 10 shows that the communication cost is large in agents distribution type than environment one but more stable. That is because in flocking with agent distribution, the number of agents fixed in all machines and the information which has to be sent between machines should be in the same size too. Whereas in environment distribution, some of the birds can be together in one big group on the sky (on one machine), and other machines maybe have less birds. That should make the communication cost is lower but less stable (variant) in environment distribution. However in prey-predator model (figure 11), the stability exists in environment distribution and not in agent distribution, that is because in prey-predator model there are agents that reproduce and die during the simulation time steps in the same machines. Thus in agent distribution, it can make more (or less) charge of agents in one machine than others, and messages size can be changed during the simulation to look like a cosine function (as the number of agents is reduced and reincreased during the simulation) that can make it less stable in agent distribution than environment one.

\subsection{When Should We Use Each Type of Distributions}

Table 2 shows a comparison between the two models (prey-predator and flocking) with two distribution types (agent and environment). Environment distribution type is better in execution time for preypredator model than flocking model, whereas agents distribution type is better for flocking model than prey-predator model. However, for communication costs, environment distribution is more stable for prey-predator model than agent distribution, whereas agents distribution is more stable for flocking model than environment one. To summarize, some distribution types are more suitable for some applications than others.

To analyse this result, we try to extract some general features from both models, then the user can choose which distribution type is more suitable for his application (see table 3). In prey-predator model, agents' life-cycle is short ( $N$ TS) and agents exist overall the environment. Whereas in flocking model, it is completely opposite to that, agents' life-cycle is long and agents may be aggregated during the simulation in one place only (not everywhere). The aggregation and the long life-cycle could make the environment distribution approach is bad solution for flocking model, because computation can be aggregated in one machine only. For that, the agents distribution is the best solution for flocking model. Whereas for prey-predator, it is completely inverted.

\section{CONCLUSIONS}

In this paper, we have proposed two types of distribution for multi-agent systems: environment distribution and agents distribution. We have evaluated it with two different applications, which are the flocking behaviour in birds and prey-predator models. In case of environment distribution, the simulated environment is divided into different partitions on different machines (each partition is allocated on one machine only). During the simulation, each machine communicates with its neighbouring machines to collect a needed information about common areas (or ghostarea). In case of agents distribution, the simulated environment is the same for all machines and agents will be divided between machines. During the simulation, each machine communicates with others for collecting needed information about other agents (or ghostagents) which exist on other machines. We propose a simple protocol to allow all machines from communicate between each other to build a distributed 
Table 2: A comparison between two models with two distribution types.

\begin{tabular}{|c|c|c|}
\hline Model & Agent distribution & Environment distribution \\
\hline Prey-predator execution time & Not efficient & Efficient \\
\hline Flocking execution time & Efficient & Not efficient \\
\hline Prey-predator communication cost & Not stable & Stable \\
\hline Flocking communication cost & Stable & Not stable \\
\hline
\end{tabular}

multi-agent simulation. The main technical problem to resolve was interactions between two agents or more from different machines, which is solved by an agreement protocol. Experimental results show that the proposed distribution types have better performances in some models than others. For example, prey-predator model has better performance in the execution time than flocking model when we distribute the environment. Whereas, agents distribution type is better for flocking model. The current implementation provides a good framework for future works, we plan to investigate more the two types of distributions, and try to implement a hybrid approach, which maybe give us better performance in most models. We plan to increase the scalability of our framework. We currently reach near 5 million agents and we plan to distribute multi-billions agents in less than one minute for one simulation time step.

Table 3: Analysis of agent's features between two models: prey-predator and flocking.

\begin{tabular}{|c|c|c|}
\hline Agent Features & Prey-predator & Flocking \\
\hline Life-cycle & Short & Long \\
\hline Movement & Small area & Large area \\
\hline Positioning & Everywhere & Aggregation \\
\hline Reproducing & Exist & Not exist \\
\hline
\end{tabular}

\section{REFERENCES}

Bromuri, S. and Stathis, K. (2009). Distributed agent environments in the ambient event calculus. In Proc.of $D E B S$, pages 12:1-12:12, New York, USA. ACM.

Cordasco, G., Rosario, D. C., Ada, M., Dario, M., Vittorio, S., and Carmine, S. (2011). A framework for distributing agent-based simulations. In Proc. of HeteroPar2011. Springer Berlin Heidelberg.

Cosenza, B., Cordasco, G., De Chiara, R., and Scarano, V. (2011). Distributed load balancing for parallel agentbased simulations. In PDP.

Isenburg, M., Lindstrom, P., and Childs, H. (2010). Parallel and streaming generation of ghost data for structured grids. CGA, IEEE, 30(3):32 - 44 .

Jefferson, D. R. (1985). Virtual time. ACM Trans. Program. Lang. Syst., 7:404-425.

Kuhl, F., Weatherly, R., and Dahmann, J. (1999). Creating computer simulation systems: an introduction to the high level architecture. Prentice Hall PTR, NJ, USA.
Logan, B. and Theodoropoulos, G. (2001). The distributed simulation of multiagent systems. Proceedings of the IEEE, 89(2):174 -185.

Miyata, N. and Ishida, T. (2008). Community-based load balancing for massively multi-agent systems. In Massively Multi-Agent Technology, volume 5043 of LNCS, pages 28-42. Springer Berlin / Heidelberg.

Motshegwa, T. and Schroeder, M. (2004). Interaction monitoring and termination detection for agent societies: Preliminary results. In ESAW, volume 3071 of $L N C S$, pages 519-519. Springer Berlin / Heidelberg.

Reynolds, C. (1999). Steering behaviors for autonomous characters.

Russell, S. J. and Norvig, P. (1996). Artificial intelligence: a modern approach. Prentice-Hall.

Scerri, D., Drogoul, A., Hickmott, S., and Padgham, L. (2010). An architecture for modular distributed simulation with agent-based models. In AAMAS'10 Proceedings., pages 541-548.

Siebert, J., Ciarletta, L., and Chevrier, V. (2010). Agents and artefacts for multiple models co-evolution: building complex system simulation as a set of interacting models. In Proceedings of the 9th Int. Conf. on AAMS, AAMAS '10, pages 509-516, Richland, SC. IFAAMAS.

Šišlák, D., Volf, P., Jakob, M., and Pěchouček, M. (2009). Distributed platform for large-scale agent-based simulations. In Agents for Games and Simulations, pages 16-32. Springer-Verlag, Berlin.

Wilensky, U. (1997). Netlogo wolf-sheep predation model.

Wooldridge, M. and Jennings, N. R. (1995). Intelligent agents: theory and practice. The Knowledge Engineering Review, 10:115-152.

Yamamoto, G., Tai, H., and Mizuta, H. (2008). A platform for massive agent-based simulation and its evaluation. In Jamali, N., Scerri, P., and Sugawara, T., editors, Massively Multi-Agent Technology, volume 5043, pages 1-12. Springer Berlin Heidelberg. 